\title{
Study on potato consumption will increase confusion regarding food and the risk of gestational diabetes
}

\author{
Patrick Mullie professor ${ }^{12}$, Mathieu Boniol professor ${ }^{13}$, Philippe Autier professor $^{13}$ \\ ${ }^{1}$ International Prevention Research Institute, 95 Cours Lafayette, 69006 Lyon, France; ${ }^{2}$ Vrije Universiteit Brussel, Elsene, Belgium; ${ }^{3}$ Strathclyde \\ University Global Public Health Institute, Lyon, France
}

Using data from the Nurses' Health Study II, Bao and colleagues found a higher prevalence of gestational diabetes mellitus (GDM) with increasing potato consumption. ${ }^{1}$ The authors stated that the high glycaemic index of potatoes, which causes a sharp postprandial rise in blood glucose concentrations and a risk of associated pancreatic $\beta$ cells exhaustion, could explain this association.

However, use of the glycaemic index has methodological limitations. Dodd and colleagues found that the glycaemic index of a meal is overestimated by $22-50 \%$ when a formula is used rather than direct measurement. ${ }^{2}$ This overestimation was unpredictable and food dependent-for example, it was greater for spaghetti than for potato. Glycaemic index therefore has low reproducibility, because potatoes are almost always consumed during meals.

It is not clear how Bao and colleagues calculated glycaemic index and glycaemic load - there is no information in the method section. ${ }^{1}$ It is also not clear why they chose potatoes to test this glycaemic index hypothesis. The glycaemic index of potatoes ranges from 60 to 120, depending of the cooking method and the type of potato consumed. It would have been easier to use a more homogenous food group, such as breakfast cereals, which have a glycaemic index of 100-120.

Bao and colleagues found that the adjusted relative risk for GDM associated with high consumption of baked, boiled, or mashed potatoes before pregnancy was 1.52 (95\% CI 1.11 to 2.07), and for French fries 1.18 (0.91 to 1.53). This discrepancy is difficult to explain using the glycaemic index theory, because the glycaemic index of French fries is higher than that of baked, boiled, or mashed potatoes.

As indicated in the paper's table 1, the mean (SD) glycaemic index and glycaemic load for the total dietary pattern across the weekly consumption servings of potatoes remained stable, ranging from 53.1 (3.7) to 55.6 (2.7) and 124.4 (22.9) to 124.1 (20.2), respectively.
As the authors pointed out, women with higher potato consumption had a higher prevalence of smoking, adiposity, and family history of diabetes, and they were less physically active. They also had a lower score for the Alternate Healthy Eating Index 2010, indicating a less healthy dietary pattern. Mean (SD) BMI for low and high pre-pregnancy potato consumers were $22.9(4.0) \mathrm{kg} / \mathrm{m}^{2}$ and $24.2(4.8) \mathrm{kg} / \mathrm{m}^{2}$. The mean energy intake was $2049 \mathrm{kcal} / \mathrm{day}$ versus $2249 \mathrm{kcal} /$ day $(1$ $\mathrm{kcal}=4.18 \mathrm{~kJ}$ ), respectively. Unfortunately, there was no information about added sugar consumption across the potato consumption groups.

The authors observed a clustering of unhealthy behaviours associated with high potato consumption. Clustering makes it difficult to single out specific contributions because even after adjustment residual confounding may remain, owing to inaccurate measurements of variables, and even after hypothetical perfect measurement, the risk of multicollinearity is likely to threaten the correct interpretation of multivariate models.

In conclusion, we believe that this reductionist approach will add to the confusion regarding food and GDM. Moreover, this approach will create inappropriate nutritional certitudes by exaggerating the food scientists' knowledge about the association between specific foods and health. Fetishising foods on the basis of nutrient composition shifts the focus to details and ignores the essentials of staying healthy-a plant based dietary pattern, not smoking, maintaining a healthy weight, and physical activity.

\section{Competing interests: None declared.}

Bao W, Tobias DK, Hu FB, Chavarro JE, Zhang C. Pre-pregnancy potato consumption and risk of gestational diabetes mellitus: prospective cohort study. BMJ 2016:352:h6898. doi:10.1136/bmj.h6898. 26759275.

2 Dodd $\mathrm{H}$, Williams S, Brown R, Venn B. Calculating meal glycemic index by using measured and published food values compared with directly measured meal glycemic index. Am J Clin Nutr 2011;94:992-6. doi:10.3945/ajcn.111.012138. 21831990.

3 Foster-Powell K, Holt SH, Brand-Miller JC. International table of glycemic index and glycemic load values: 2002. Am J Clin Nutr 2002;76:5-56.12081815. 
Published by the BMJ Publishing Group Limited. For permission to use (where not already permissions granted under a licence) please go to http://group.bmj.com/group/rights-licensing 